\title{
Queixa de Zumbido e Alterações de Saúde
}

\section{Tinnitus Complaint and Health Changes}

\section{Queja del Acúfeno y Alteraciones de la Salud}

\author{
Geise Corrêa Ferreira* \\ Lidiéli Dalla Costa* \\ Maryndia Diehl Muller* \\ Maristela Julio Costa*
}

\section{Resumo}

Objetivo: Verificar a relação entre a queixa de zumbido, em pacientes com rebaixamento dos limiares das frequências a partir de $3000 \mathrm{~Hz}$, com o gênero e faixa etária e associação com alterações de saúde. Métodos: Foram analisados os prontuários de adultos e idosos, atendidos entre o período de setembro de 2013 a junho de 2016, com queixa de zumbido e diagnóstico audiológico de audição normal considerando a média das frequências de 500,1000 e $2000 \mathrm{~Hz}$, com rebaixamento nas frequências a partir de $3000 \mathrm{~Hz}$ em ambas as orelhas. Considerou-se na análise, a ausência ou presença de alteração de saúde autorrelatadas como hipertensão, diabetes, colesterol e alterações psicossociais como depressão e ansiedade. Resultados: O grupo estudado ficou constituído por 38 sujeitos, 21 mulheres e 17 homens, sendo 21 adultos e 17 idosos. Do total, 68,4\% referiram alteração de saúde, desses, 50\% relataram hipertensão arterial, 18,4\% alteração psicossocial e 13,2\% diabetes e colesterol. Existiu associação significativa entre a presença de alteração de saúde e as mulheres, bem como entre a variável hipertensão arterial e as mulheres idosas. Conclusão: Encontrou-se distribuição semelhante tanto na variável gênero como na idade, com discreta prevalência das mulheres em relação aos homens, e dos adultos com relação aos idosos. A maioria dos pacientes $(68,4 \%)$ apresentou alguma alteração de saúde, havendo prevalência de alterações nas mulheres. Dentre as alterações, a Hipertensão Arterial foi a mais referida (50\%), com associação significativa nas mulheres idosas.

Palavras-chave: Zumbido; Audição; Doença; Autorrelato; Adulto; Idoso; Perda Auditiva

*Universidade Federal de Santa Maria, Santa Maria, RS, Brasil.

Contribuição dos autores:

GCF: delineamento do estudo, coleta, análise, interpretação dos resultados, redação e revisão final. LDC: análise e interpretação dos resultados, revisão e aprovação final. MDM: concepção do estudo e coleta de dados, revisão final. MJC: delineamento do estudo, análise e interpretação dos resultados, redação e revisão final.

E-mail para correspondência: Geise Corrêa Ferreira - geisecorrea@gmail.com Recebido: $16 / 12 / 2016$

Aprovado: $11 / 06 / 2017$ 


\section{Abstract}

Purpose: To verify the relation between tinnitus complaint, in patients with lower frequency thresholds from $3000 \mathrm{~Hz}$, with gender and age group and association with health changes. Methods: The medical records of adults and the elderly were analyzed. They were treated in the period between September 2013 and June 2016, with complaints of tinnitus and audiological diagnosis of normal hearing, considering the means of frequencies of 500, 1000 and $2000 \mathrm{~Hz}$, with lowering frequency of $3000 \mathrm{~Hz}$ in both ears. The analysis included the absence or presence of self-reported health disorders such as hypertension, diabetes, cholesterol, and psychosocial disorders such as depression and anxiety. Results: The study group consisted of 38 patients, 21 women and 17 men, 21 adults and 17 elderly people. From the total, $68.4 \%$ reported health changes, $50 \%$ reported hypertension, $18.4 \%$ presented psychosocial changes, and $13.2 \%$ had diabetes and cholesterol. There was a significant association between the presence of altered health in women, as well as variable hypertension in elderly women. Conclusion: A similar distribution was found in both gender and age variable, with a low prevalence in women compared to men, and in adults compared to the elderly people. Most of the patients $(68,4 \%)$ presented some health change, with prevalence of changes in women. Among changes, Arterial Hypertension was the most referred $(50 \%)$, with a significant association regarding elderly women.

Keywords: Tinnitus; Hearing; Disease; Self Report; Adult; Aged; Hearing Loss

\section{Resumen}

Objetivo: Verificar la relación entre la queja del acúfeno, en pacientes con reducción de los umbrales de frecuencias comprendidos entre $3000 \mathrm{~Hz}$, con el género y la edad y asociación con alteraciones de salud. Métodos: Fueron analizados los registros de adultos y ancianos, atendidos entre el período de septiembre de 2013 a junio de 2016, con queja de acúfeno y diagnóstico audiológico de audición normal considerando la Media Tritonal de 500, 1000 y $2000 \mathrm{~Hz}$, con reducción en las frecuencias a partir de $3000 \mathrm{~Hz}$ en ambas orejas. Se Consideró en el análisis, la ausencia o presencia de alteraciones de salud autorrelatadas como hipertensión, diabetes, colesterol y alteraciones psicosociales como depresión, ansiedad. Resultados: El grupo estudiado quedó constituido por 38 individuos, 21 mujeres y 17 hombres, siendo 21 adultos y 17 ancianos. Del total $68,4 \%$ presentaron alteraciones de salud, de esos, 50\% presentaron hipertensión arterial, 18,4\% alteración psicosocial y 13,2\% alteración de diabetes y de colesterol. Existió asociación significativa entre la presencia de alteración de salud de las mujeres, bien como entre a variable hipertensión arterial y las mujeres ancianas. Conclusión: Se encontro distribuición semejante tanto en el variable género como edad, con discreta prevalencia de las mujeres en relación a los hombres y de los adultos con relación a los ancianos. La mayoría de los pacientes presentó alguna alteración de salud $(68,4 \%)$, hubo prevalencia de alteraciones en las mujeres. Entre las alteraciones, la Hipertensión Arterial fue la más citada (50\%), con asociación significativa en las mujeres ancianas.

Palabras clave: Acúfeno; Audición; Enfermedad; Autoinforme; Adulto; Anciano; Pérdida Auditiva

\section{Introdução}

Descrito como a percepção de um som na ausência de uma fonte geradora externa correspondente ${ }^{1}$, o zumbido vem sendo uma queixa otológica de grande ocorrência na clínica fonoaudiológica. Segundo dados internacionais, a prevalência do zumbido na população geral subiu de $15 \%$ para $25,3 \%$ em apenas 15 anos, sendo que esses números crescem com o avanço da idade ${ }^{2}$. Com base em estudo epidemiológico nacional ${ }^{3}$, a prevalência do zumbido corresponde a $22 \%$ na população adulta do município de São Paulo.

Considerado como um sintoma que acomete as vias auditivas, o zumbido pode ter como causas doenças otológicas, como trauma acústico, exposição ao ruído, presbiacusia, entre outras ${ }^{3,4}$. Podendo ser decorrente, ainda, de doenças que afetam secundariamente o ouvido, como as alterações metabólicas, cardiovasculares, neurológicas, farmacológicas, psiquiátricas e odontológicas ${ }^{3,4}$. 
A Hipertensão Arterial (HA) é um distúrbio circulatório, considerado crônico, assintomático e de origem multifatorial, caracterizado pela presença de elevados níveis de pressão sanguínea ${ }^{5}$. No Brasil, estima-se que mais de $30 \%$ da população seja hipertensa, podendo chegar a mais de $50 \%$ após os 60 anos $^{6}$. Nesta população, o zumbido é um sintoma comumente referido ${ }^{7,8}$.

Com relação às alterações metabólicas, a prevalência do Diabetes Mellitus (DM) vem crescendo nos últimos anos ${ }^{9}$. Ainda segundo os autores, os sintomas de hipoacusia, zumbido e tontura são frequentemente referidos por indivíduos diabéticos. A queixa de zumbido também vem sendo evidenciada em pacientes com alteração de colesterol ${ }^{10}$.

Estudos demonstram que o zumbido pode ocasionar prejuízo na qualidade de vida, interferindo no trabalho, no sono e na comunicação ${ }^{11,12}$. Muitas vezes, sua percepção excede a capacidade do indivíduo de adaptação e tolerância, podendo causar assim esgotamento físico, mental e/ou emocional ${ }^{11}$.

A queixa de zumbido está relacionada a aspectos psicológicos, os quais interferem no modo como o zumbido será interpretado e abordado pelo indivíduo ${ }^{13}$. Desta maneira, as reações emocionais atribuídas ao zumbido são consideradas determinantes ao desconforto provocado pelo mesmo ${ }^{14}$, podendo este estar associado à depressão, ansiedade e outras desordens psicológicas e psiquiátricas ${ }^{1}$.

A presença da queixa de zumbido vem constantemente sendo evidenciada em indivíduos sem ou com perda auditiva ${ }^{2,15,16}$. Nos casos com zumbido associado à perda auditiva, recomenda-se o uso de próteses auditivas, como forma de intervenção, na busca da diminuição dos efeitos que a deficiência auditiva provoca na qualidade de vida, para minimizar ou eliminar a percepção do zumbido, bem como na redução das dificuldades de reconhecimento de fala por eles apresentadas. Entretanto, em casos nos quais o rebaixamento de limiares é restrito às frequências altas, a indicação de próteses auditivas é considerada controversa de acordo com a Portaria GM 793 e 835 (2012) ${ }^{17}$. Em clínicas privadas, esse tipo de indicação também é questionado, uma vez que o benefício esperado com o uso da amplificação sonora nem sempre é alcançado.

Portanto, conforme exposto, percebe-se que a intervenção frente ao zumbido é um desafio, principalmente nos casos dos pacientes com configurações auditivas que geram controvérsia com relação à adaptação de próteses auditivas. Nestes casos, torna-se ainda mais importante as investigações de quais fatores podem estar associados a esse sintoma, na tentativa de amenizar o desconforto com o mesmo.

Com isso, devido à necessidade de esclarecer aspectos referentes à fisiopatologia do zumbido, buscando auxiliar no tratamento do mesmo, objetivou-se por meio deste estudo, verificar a relação entre a queixa de zumbido, em pacientes com rebaixamento dos limiares das frequências a partir de $3000 \mathrm{~Hz}$, com o gênero e a faixa etária, bem como a associação entre a queixa de zumbido e alterações de saúde referidas.

\section{Método}

Trata-se de um estudo retrospectivo realizado por meio de análise de banco de dados e levantamento dos pacientes com queixa de zumbido, que realizaram avaliação audiológica entre o período de setembro de 2013 a junho de 2016. $\mathrm{O}$ estudo foi desenvolvido em um Serviço de Atenção à Saúde Auditiva de uma Instituição de Ensino Superior, previamente aprovado pelo Comitê de Ética em Pesquisa, sob o protocolo de $n^{\circ}$ 05765712.3.0000.5346.

Foram analisados os prontuários de adultos e idosos, considerando como critérios de inclusão: presença de queixa de zumbido e diagnóstico audiológico de audição normal considerando-se a média tritonal das frequências de 500, 1000 e $2000 \mathrm{~Hz}$, com rebaixamento (a partir de $25 \mathrm{~dB}$ ) nos limiares das frequências altas em ambas as orelhas (a partir de $3000 \mathrm{~Hz})^{18,19}$.

Considerou-se na análise, a ausência ou presença de alterações de saúde como hipertensão, diabetes (não sendo especificado o tipo), colesterol, bem como alterações psicossociais como depressão, ansiedade, sendo que estas foram autorreferidas pelos indivíduos durante a realização da anamnese, sem a comprovação médica do diagnóstico ou da hipótese diagnóstica. Consideraram-se como presentes as alterações psicossociais, quando mencionado no prontuário o uso de medicação para intervenção. Para análise da faixa etária, os pacientes foram reunidos em dois grupos conforme a idade: adultos, na faixa etária entre 30 e 60 anos e idosos, com idade acima de 60 anos.

Foram excluídos aqueles indivíduos cujas informações relacionadas à queixa de zumbido e/ ou problemas de saúde estavam incompletas, que 
apresentaram alterações de orelha média e perda auditiva do tipo condutiva e/ou mista, assim como aqueles que apresentaram ou referiram alterações neurológicas.

Para definir a amostra do estudo, inicialmente realizou-se a análise descritiva das variáveis. Para o processamento dos dados utilizou-se o Microsoft Office Excel. $\mathrm{Na}$ análise estatística utilizou-se o software SPSS (Statistical Package For Social Sciences) versão 15.0. Na análise bivariada foram feitos testes de associação pelo Qui-quadrado e teste Exato de Fischer quando procedente, devido à quantidade de caselas esperadas ser inferior a $5 \%$. Para a análise da comparação entre a presença das alterações e ausência em relação a variável idade, inicialmente empregou-se o teste de normalidade Kolmogorov-Smirnov e a distribuição foi considerada normal, pois $\mathrm{p}>0,05$.

\section{Resultados}

A amostra estudada constituiu-se de 38 pacientes, com queixa de zumbido e rebaixamento dos limiares a partir da frequência de $3000 \mathrm{~Hz}$, sendo 26 pacientes, com alteração de saúde e 12 sem alterações de saúde. Desses, 21 eram do gênero feminino e 17 do gênero masculino, com idades variando de 34 a 70 anos. A média geral de idade foi de 55,95 anos $( \pm 9,26)$, sendo do gênero feminino de 53,71 $( \pm 9,20)$ anos e do masculino de $58,71( \pm 8,81)$ anos. O grupo de idosos foi formado por 17 sujeitos, com média de $64,35( \pm 3,04)$ anos e o de adultos, por 21 sujeitos, com média de idade de $49,14( \pm 6,52)$ anos.

Na tabela 1 estão expostos os dados descritivos referentes ao grupo amostral, considerando as variáveis gênero, grupos segundo a faixa etária e as alterações de saúde.

Tabela 1. Características dos indivíduos com queixa de zumbido e rebaixamento dos limiares a partir da frequência de $3000 \mathrm{~Hz}$

\begin{tabular}{ccc}
\hline Variáveis & Frequência & Percentual \\
Gênero & 17 & $44,7 \%$ \\
Masculino & 21 & $55,3 \%$ \\
Feminino & 21 & $55,3 \%$ \\
Grupos & 17 & $44,7 \%$ \\
Adultos & & \\
Idosos & 19 & $50,0 \%$ \\
Hipertensão & 19 & $50,0 \%$ \\
Presente & & \\
Ausente & 5 & $13,2 \%$ \\
Diabetes & 33 & $86,8 \%$ \\
Presente & & \\
Ausente & 5 & $13,2 \%$ \\
Colesterol & 33 & $86,8 \%$ \\
Presente & & \\
Ausente & 7 & $18,4 \%$ \\
Psicossocial & 31 & $81,6 \%$ \\
Presente & & \\
Ausente & &
\end{tabular}


Tabela 2. Avaliação da relação do gênero com as alterações de saúde autorrelatadas

\begin{tabular}{|c|c|c|c|c|}
\hline \multirow[b]{2}{*}{ Variáveis } & \multirow[b]{2}{*}{ Total } & \multicolumn{2}{|c|}{ Gênero } & \multirow[b]{2}{*}{ p-valor } \\
\hline & & $\begin{array}{c}\text { Masculino } \\
\text { n (\%) }\end{array}$ & $\begin{array}{c}\text { Feminino } \\
\text { n }(\%)\end{array}$ & \\
\hline \multicolumn{5}{|c|}{ Hipertensão } \\
\hline Presente & $19(50 \%)$ & $5(29,4 \%)$ & $14(66,7 \%)$ & \\
\hline Ausente & $19(50 \%)$ & $12(70,6 \%)$ & $7(33,3 \%)$ & $0,022 *$ \\
\hline \multicolumn{5}{|c|}{ Diabetes } \\
\hline Presente & $5(13,2 \%)$ & $1(5,9 \%)$ & $4(19,0 \%)$ & \\
\hline Ausente & $33(86,8 \%)$ & $16(94,1 \%)$ & $17(81,0 \%)$ & $0,243 \mathrm{~F}$ \\
\hline \multicolumn{5}{|c|}{ Colesterol } \\
\hline Presente & $5(13,2 \%)$ & $2(11,8 \%)$ & $3(14,3 \%)$ & \\
\hline Ausente & $33(86,8 \%)$ & $15(88,2 \%)$ & $18(85,7 \%)$ & $0,604 \mathrm{~F}$ \\
\hline \multicolumn{5}{|c|}{ Psicossocial } \\
\hline Presente & $7(18,4 \%)$ & $1(5,9 \%)$ & $6(28,6 \%)$ & \\
\hline Ausente & $31(81,6 \%)$ & $16(94,1 \%)$ & $15(71,4 \%)$ & $0,082 \mathrm{~F}$ \\
\hline \multicolumn{5}{|c|}{ Alterações } \\
\hline Presente & $26(68,4 \%)$ & $8(47,1 \%)$ & $18(85,7 \%)$ & \\
\hline Ausente & $12(31,6 \%)$ & $9(52,9 \%)$ & $3(14,3 \%)$ & $0,011 *$ \\
\hline
\end{tabular}

Teste Qui-Quadrado $\mathrm{e}^{\mathrm{F}}$ teste exato de Fischer.

*Valores com associação significativamente $(p \leq 0,05)$

Verificou-se a existência de associação entre o gênero e a hipertensão, sendo que o gênero feminino está associado à presença de hipertensão. Outra variável associada significativamente ao sexo foi a presença ou não de alterações, sendo que as mulheres têm maior prevalência de presença de alterações de saúde.

Tabela 3. Avaliação da relação dos grupos de faixa etária com as alterações de saúde

\begin{tabular}{|c|c|c|c|c|}
\hline \multirow[b]{2}{*}{ Variáveis } & \multirow[b]{2}{*}{ Total } & \multicolumn{2}{|c|}{ Grupo } & \multirow[b]{2}{*}{ p-valor } \\
\hline & & $\begin{array}{l}\text { Adulto } \\
\text { n (\%) }\end{array}$ & $\begin{array}{l}\text { Idoso } \\
\text { n (\%) }\end{array}$ & \\
\hline \multicolumn{5}{|c|}{ Hipertensão } \\
\hline Presente & $19(50 \%)$ & $11(52,4 \%)$ & $8(47,1 \%)$ & \multirow{2}{*}{0,744} \\
\hline Ausente & $19(50 \%)$ & $10(47,6 \%)$ & $9(52,9 \%)$ & \\
\hline \multicolumn{5}{|c|}{ Diabetes } \\
\hline Presente & $5(13,2 \%)$ & $4(19,0 \%)$ & $1(5,9 \%)$ & \multirow{2}{*}{$0,243 \mathrm{~F}$} \\
\hline Ausente & $33(86,8 \%)$ & $17(81,0 \%)$ & $16(94,1 \%)$ & \\
\hline \multicolumn{5}{|c|}{ Colesterol } \\
\hline Presente & $5(13,2 \%)$ & $1(4,8 \%)$ & $4(23,5 \%)$ & \multirow{2}{*}{$0,112 \mathrm{~F}$} \\
\hline Ausente & $33(86,8 \%)$ & $20(95,2 \%)$ & $13(76,5 \%)$ & \\
\hline \multicolumn{5}{|c|}{ Psicossocial } \\
\hline Presente & $7(18,4 \%)$ & $5(23,8 \%)$ & $2(11,8 \%)$ & \multirow{2}{*}{$0,302 \mathrm{~F}$} \\
\hline Ausente & $31(81,6 \%)$ & $16(76,2 \%)$ & $15(88,2 \%)$ & \\
\hline \multicolumn{5}{|c|}{ Alterações } \\
\hline Presente & $26(68,4 \%)$ & $14(66,7 \%)$ & $12(70,6 \%)$ & \multirow{2}{*}{0,796} \\
\hline Ausente & $12(31,6 \%)$ & $7(33,3 \%)$ & $5(29,4 \%)$ & \\
\hline
\end{tabular}

Teste Qui-Quadrado e Fteste Exato de Fischer.

*Valores com associação significativamente $(p \leq 0,05)$ 
Com base nos resultados, não houve associação significativa com relação às alterações de saúde analisadas segundo a variável idade (adulto ou idoso).
Não ocorreu associação significativa entre a variável gênero e nenhuma das alterações de saúde estudadas no grupo de adultos.

Tabela 4. Avaliação da relação entre gênero e as alterações de saúde do grupo de adultos.

\begin{tabular}{|c|c|c|c|c|}
\hline \multirow[b]{2}{*}{ Variáveis } & \multirow[b]{2}{*}{ Total } & \multicolumn{2}{|c|}{ Gênero } & \multirow[b]{2}{*}{ p-valor } \\
\hline & & $\begin{array}{c}\text { Masculino } \\
\text { n (\%) }\end{array}$ & $\begin{array}{c}\text { Feminino } \\
\text { n (\%) }\end{array}$ & \\
\hline \multicolumn{5}{|c|}{ Hipertensão } \\
\hline Presente & $11(52,4 \%)$ & $2(33,3 \%)$ & $9(60,0 \%)$ & \multirow{2}{*}{$0,268 \mathrm{~F}$} \\
\hline Ausente & $10(47,6 \%)$ & $4(66,7 \%)$ & $6(40,0 \%)$ & \\
\hline \multicolumn{5}{|c|}{ Diabetes } \\
\hline Presente & $4(19,0 \%)$ & $0(0,0 \%)$ & $4(26,7 \%)$ & \multirow{2}{*}{$0,228 \mathrm{~F}$} \\
\hline Ausente & $17(81,0 \%)$ & $6(100,0 \%)$ & $11(73,3 \%)$ & \\
\hline \multicolumn{5}{|c|}{ Colesterol } \\
\hline Presente & $1(4,8 \%)$ & $0(0,0 \%)$ & $1(6,7 \%)$ & \multirow{2}{*}{$0,714 \mathrm{~F}$} \\
\hline Ausente & $20(95,2 \%)$ & $6(100,0 \%)$ & $14(93,3 \%)$ & \\
\hline \multicolumn{5}{|c|}{ Psicossocial } \\
\hline Presente & $5(23,8 \%)$ & $0(0,0 \%)$ & $5(23,8 \%)$ & \multirow{2}{*}{$0,148 \mathrm{~F}$} \\
\hline Ausente & $16(76,2 \%)$ & $6(100,0 \%)$ & $10(76,2 \%)$ & \\
\hline \multicolumn{5}{|c|}{ Alterações } \\
\hline Presente & $14(66,7 \%)$ & $2(33,3 \%)$ & $12(80,0 \%)$ & \multirow{2}{*}{$0,064 \mathrm{~F}$} \\
\hline Ausente & $7(33,3 \%)$ & $4(66,7 \%)$ & $3(20,0 \%)$ & \\
\hline
\end{tabular}

Teste Qui-Quadrado e Fteste Exato de Fischer

*Valores com associação significativamente $(p \leq 0,05)$

Tabela 5. Avaliação da relação entre gênero e as alterações do grupo de idosos

\begin{tabular}{|c|c|c|c|c|}
\hline \multirow[b]{2}{*}{ Variáveis } & \multirow[b]{2}{*}{ Total } & \multicolumn{2}{|c|}{ Gênero } & \multirow[b]{2}{*}{ p-valor } \\
\hline & & $\begin{array}{c}\text { Masculino } \\
\text { n (\%) }\end{array}$ & $\begin{array}{c}\text { Feminino } \\
\text { n (\%) }\end{array}$ & \\
\hline \multicolumn{5}{|c|}{ Hipertensão } \\
\hline Presente & $8(47,1 \%)$ & $3(27,3 \%)$ & $5(83,3 \%)$ & \\
\hline Ausente & $9(52,9 \%)$ & $8(72,7 \%)$ & $1(16,7 \%)$ & $0,043 F^{*}$ \\
\hline \multicolumn{5}{|c|}{ Diabetes } \\
\hline Presente & $1(5,9 \%)$ & $1(9,1 \%)$ & $0(0,0 \%)$ & \\
\hline Ausente & $16(94,1 \%)$ & $10(90,9 \%)$ & $6(100,0 \%)$ & $0,647 \mathrm{~F}$ \\
\hline \multicolumn{5}{|c|}{ Colesterol } \\
\hline Presente & $4(23,5 \%)$ & $2(18,2 \%)$ & $2(33,3 \%)$ & \\
\hline Ausente & $13(76,5 \%)$ & $9(81,8 \%)$ & $4(66,7 \%)$ & $0,445 \mathrm{~F}$ \\
\hline \multicolumn{5}{|c|}{ Psicossocial } \\
\hline Presente & $2(11,8 \%)$ & $1(9,1 \%)$ & $1(16,7 \%)$ & \\
\hline Ausente & $15(88,2 \%)$ & $10(90,0 \%)$ & $5(83,3 \%)$ & $0,596 \mathrm{~F}$ \\
\hline \multicolumn{5}{|c|}{ Alterações } \\
\hline Presente & $12(70,6 \%)$ & $6(54,5 \%)$ & $6(100,0 \%)$ & \\
\hline Ausente & $5(29,4 \%)$ & $5(45,5 \%)$ & $0,0(0,0 \%)$ & $0,075 \mathrm{~F}$ \\
\hline
\end{tabular}

Teste Qui-Quadrado e Fteste Exato de Fischer.

*Valores com associação significativamente $(p \leq 0,05)$ 
Encontrou-se associação significativa entre gênero e hipertensão, sendo que o gênero feminino apresentou maior prevalência dessa alteração de saúde do que o gênero masculino no grupo de idosos.

\section{Discussão}

$\mathrm{Na}$ amostra estudada verificou-se que 55,5\% dos pacientes eram do gênero feminino, semelhante ao encontrado em outros estudos ${ }^{3,7,15,20}$ (Tabela 1). Com relação à influência do gênero no predomínio da queixa de zumbido, os achados na literatura são controversos, tendo sido verificado em alguns estudos a prevalência do gênero masculino ${ }^{21} \mathrm{e} \mathrm{em}$ outros do gênero feminino ${ }^{7,22}$. Entretanto, dificilmente as diferenças entre os gêneros são estatisticamente significativas.

A prevalência da queixa de zumbido teve um aumento significativo nos últimos 15 anos, indo de $15 \%$ para $25,3 \%$ na população em geral ${ }^{2}$, sendo verificada sua ocorrência em todas as idades ${ }^{3}$. A literatura aponta, ainda, que o crescimento da prevalência do zumbido ocorre com o aumento da idade ${ }^{1,3,15}$.

Com relação às faixas etárias estabelecidas, constatou-se que o grupo de adultos prevaleceu em relação aos idosos (Tabela 1). Tal fato pode ser associado ao perfil audiológico da amostra, o qual apresentava rebaixamento de limiares auditivos apenas nas frequências altas, indicando o início da instalação da perda auditiva. Assim sendo, acredita-se que houve menos idosos nesta análise, pois, à medida que ocorre a progressão da idade, há uma tendência de comprometimento também das frequências médias e/ou baixas ${ }^{23,24}$.

Corroborando com esse achado, em uma pesquisa realizada com pacientes diabéticos e não diabéticos com idade de 33 a 84 anos, ao considerar a perda auditiva, relacionada à idade, os autores observaram prevalência de limiares auditivos dentro dos padrões de normalidade com prejuízo restrito às frequências altas, enquanto nas idades superiores, observaram alterações neurossensoriais instaladas em outras regiões de frequências ${ }^{23}$.

$\mathrm{O}$ zumbido vem sendo investigado por diferentes áreas devido à sua vasta associação a distintas patologias. Com relação às alterações de saúde analisadas, verificou-se que $68,4 \%$ da amostra apresentavam algumas das alterações estudadas (Tabela 1).
Dentre as alterações de saúde, a Hipertensão Arterial foi a principal alteração relatada pelos participantes do estudo, seguida de alterações psicossociais (Tabela 1). Pode-se observar a ocorrência de associação significativa entre o gênero feminino e a presença de alterações de saúde (Tabela 2). Achado que corrobora com o verificado em outros estudos $^{10,20,25}$.

A percepção do zumbido vem sendo apontada na literatura como um dos sintomas relatados por indivíduos hipertensos de diferentes faixas etá$\operatorname{rias}^{2,7,8}$. Em decorrência do comprometimento do aparelho circulatório, o funcionamento da orelha interna pode ser prejudicado devido à diminuição do fluxo sanguíneo capilar e do transporte de oxigênio causado pelo aumento da viscosidade sanguínea ${ }^{25}$.

A Hipertensão Arterial como fator implicado na geração do zumbido é ainda controversa. Vários estudos têm buscado analisar a associação entre alterações no aparelho auditivo e Hipertensão Arterial em populações de idades variadas, sendo que alguns autores têm verificado tal associação ${ }^{26}$ e outros não encontraram associação $0^{1,7,25}$.

Verificou-se, ainda, associação entre o gênero feminino e a Hipertensão Arterial (66,7\%) (Tabela 2 ), achado semelhante ao verificado em estudo ${ }^{25}$ que também constatou maior proporção de mulheres hipertensas com queixa de zumbido. Essa associação vai ao encontro dos dados referidos na literatura, em que a prevalência de mulheres hipertensas é maior a partir da quinta década de vida ${ }^{27}$, sendo a média de idade do gênero feminino neste estudo de 53,71 anos. A prevalência de mulheres hipertensas foi verificada em ambos os grupos da amostra, onde se verificou a existência de associação significativa entre o grupo das mulheres idosas e HA (Tabela 4 e 5).

Essa tendência pode estar relacionada às diversas alterações hormonais decorrentes do climatério e menopausa, o que acaba fragilizando o sistema cardiovascular ${ }^{27}$.

Por sua vez, as alterações de ordem psicossociais também vêm sendo evidenciadas em pacientes que apresentam percepção do zumbido ${ }^{1,2,12}$ corroborando com os achados deste estudo, no qual foi a segunda alteração de saúde mais referenciada pelos participantes (Tabela 1). Em estudo de revisão ${ }^{12}$ constatou-se alta prevalência de sintomas depressivos em indivíduos com queixa de zumbido. 
O zumbido quando classificado como fato importante pelo indivíduo, acarreta maior foco atencional, fazendo com que sua percepção seja mais evidente. Assim, sua associação com situações desagradáveis, ou até mesmo de perigo, contribuem para ativação dos centros de percepção ${ }^{28}$. Quando tal associação ocorre, o sistema límbico é ativado. Ao ativar o Sistema Límbico, responsável pelas emoções, memória e os estados de humor e de motivação, o indivíduo passa a percebê-lo por mais tempo e às vezes até mesmo com um aumento de intensidade, o que pode levar a problemas de atenção e concentração ${ }^{29}$. Pode ocorrer, também, a ativação do Sistema Nervoso Autônomo, o que pode acarretar o aumento significativo do nível de desconforto, estresse e ansiedade ${ }^{29}$.

Não foi encontrada associação significativa entre a queixa de zumbido e alterações de saúde de caráter psicossocial na amostra (Tabela 2). Esse achado discorda de outro estudo realizado com pacientes adultos a fim de se averiguar a prevalência e os fatores de riscos associados ao zumbido, no qual constataram que entre diversos fatores estão associados ao zumbido os de caráter psicológico, o estresse e a depressão ${ }^{1}$.

Apesar de não ter sido verificada associação entre o Diabetes Mellitus e Colesterol com a queixa de zumbido neste estudo, sabe-se que o metabolismo da orelha depende diretamente de fornecimento de oxigênio e glicose, assim, alterações do fluxo ou do metabolismo sanguíneo podem prejudicar o funcionamento da orelha interna ${ }^{10}$.

A alteração de colesterol, por sua vez, acarreta na diminuição da liberação do vasodilatador óxido nítrico que pode prejudicar a microcirculação da cóclea, o que acarreta a diminuição da mobilidade pelo aumento da rigidez das paredes das células ciliadas externas, prejudicando, assim, o funcionamento adequado da orelha interna ${ }^{30}$. Estudo verificou que $56,91 \%$ dos indivíduos com queixa de zumbido apresentavam hipercolesterolemia ${ }^{10}$.

Em pesquisa realizada com intuito de se verificar a sensação subjetiva do zumbido pré e pós-intervenção nutricional em pacientes com zumbido e com alterações metabólicas, foi observado que o grau de incômodo do zumbido melhorou significativamente após controle nutricional, atribuindo-se, então, que o zumbido parece ser influenciado pela dieta alimentar ${ }^{10}$.

Considera-se como limitação do estudo, o fato de não se ter investigado as alterações de saúde através de exames clínicos, as quais foram consideradas apenas pelo autorrelato do paciente, e o grupo estudado ser pequeno. Porém, com base nos resultados encontrados, acredita-se que este estudo pode servir como base para outras pesquisas, a fim de que estes achados auxiliem tanto os profissionais que realizam a indicação da prótese auditiva, como aqueles responsáveis pelo processo de adaptação da mesma, em indivíduos com tais características audiológicas.

Salienta-se a importância da investigação e da realização de intervenções quanto aos aspectos de saúde, objetivando-se minimizar os efeitos de alterações que agem secundariamente no sistema auditivo, buscando além da diminuição da degeneração do sistema, a minimização dos sintomas associados a estes.

\section{Conclusão}

Encontrou-se distribuição semelhante, tanto na variável gênero como idade, com discreta prevalência das mulheres em relação aos homens e dos adultos com relação aos idosos. A maioria dos pacientes apresentou alguma alteração de saúde, havendo prevalência de alterações nas mulheres. Dentre as alterações, a Hipertensão Arterial foi a mais frequente, com associação significativa nas mulheres idosas.

\section{Referências}

1. KIM HJ, LEE HJ, AN SY, SIM S, PARK B, KIM SW, et al. Analysis of the prevalence and associated risk factors of tinnitus in adults. 2015; 10(5): 1-15.

2. Shargorodsky J, Curhan GC, Farwell WR. Prevalence and characteristics of tinnitus among US adults. Am J Med. 2010; 123(8): 711-8

3. Oiticica J, Bittar RSM. Tinnitus prevalence in the city of São Paulo. Braz J Otorhinolaryngol. 2015; 81(2): 167-176.

4. Esteves CC, Brandão FN, Siqueira CGA; Carvalho SAS. Audição, Zumbido e Qualidade de Vida: Um Estudo Piloto. Rev CEFAC. 2012; 14(5): 836-43.

5. Lessa I. Hipertensão arterial sistêmica no Brasil: tendência temporal. Cadernos de saúde pública. 2010; 26: 470-1.

6. Sociedade Brasileira de Cardiologia, Sociedade Brasileira de Hipertensão, Sociedade Brasileira de Nefrologia. VI Diretrizes Brasileiras de Hipertensão. Arq Bras Cardiol. 2010; 95(1 supl.1): 1-51.

7. Gibrin PCD, Melo JJ, Marchiori LLM. Prevalência de queixa de zumbido e prováveis associaç̃es com perda auditiva, diabetes mellitus e hipertensão arterial em pessoas idosas. CoDAS. 2013; 25(2): 176-80. 
8. Oigman W. Sinais e sintomas em hipertensão arterial. JBM. 2014; 102(5): 13-8.

9. David LZ, Finamor MM, Buss C. Possíveis implicações audiológicas do diabetes melito: uma revisão de literatura. Rev CEFAC. 2015; 17(6): 2018-24.

10. Almeida TAS, Samelli AG, Mecca FDN, Martino E de, Paulino AM. Sensação subjetiva do zumbido pré e pós intervenção nutricional em alterações metabólicas. Pró-Fono. 2009; 21(4): 291-6.

11. Filha VAVS, Matas CG. Potenciais evocados auditivos tardios em indivíduos com queixa de zumbido. Braz J Otorhinolaryngol. 2010; 76(2): 263-70.

12. Geocze L, Mucci S, Abranches DC, Marco M A, Penido NO. Systematic review on the evidences of an association between tinnitus and depression. Braz J Otorhinolaryngol. 2013; 79(1): 106-11.

13. Santos RMR, Sanchez TG, Bento RF, Lucia MCS. Auditory hallucinations in tinnitus patients: Emotional relationships and Depression. Int Arch Otorhinolaryngol. 2012; 16(3): 322-7.

14. Holdefer L, Oliveira CACP, Venosa AR. Sucesso no tratamento do zumbido com terapia em grupo. Braz J Otorhinolaryngol. 2010; 76(1): 102-6.

15. Mondelli MFCG, Rocha AB. Correlação entre os achados audiológicos e incômodo com zumbido. Arq Int Otorrinolaringol.2011; 15(2): 172-80.

16. Urnau D, Tochetto TM. Características do zumbido e da hiperacusia em indivíduos normo-ouvintes. Arq Int Otorrinolaringol. / Intl Arch Otorhinolaryngol. 2011; 15(4): 468-74.

17. Brasil. Instrutivo Saúde Auditiva. Diretrizes para Tratamento e Reabilitação de Pessoas com Deficiência Auditiva. Portaria GM 793 de 24 de abril de 2012 e Portaria GM 835 de 25 de abril de 2012

18. Silman S, Silverman CA. Auditory diagnosis: principles and applications. San Diego: Singular Publishing Group; 1997.

19. Lloyd LL, Kaplan H. Audiometric interpretation: a manual of basic audiometry. Baltimore: University Park Press; 1978.
20. Oliveira IPC, Scharlach RC. Ocorrência de queixa de zumbido em indivíduos atendidos em uma clínica endocrinológica. Revista Equilíbrio Corporal e Saúde. 2011; $3(2): 23-32$

21. Lim HW, Kim TS, Kang WS, Song CII, Baek S, Chung JW. Effect of a 4- Week Treatment with Cilostazol in Patients with Chronic Tinnitus: A Randomized, Prospective, Placebocontrolled, Double-blind, Pilot Study. J Int Adv Otol. 2016; 12(2): 170-6.

22. Pinto PC, Sanchez TG, Tomita S. Avaliação da relação entre severidade do zumbido e perda auditiva, sexo e idade do paciente. Braz J Otorhinolaryngol. 2010; 76(1): 18-24.

23. Marchiori LLM, Gibrin, PCD. Diabetes Mellitus: Prevalência de Alterações Auditivas. Arq Bras Endocrinol Metab. 2003; 47(1): 82-6.

24. Araujo TM, Iório MCM. Perfil populacional de idosos encaminhados à seleção de próteses auditivas em hospital público. Audiol Commun Res. 2014; 19(1): 45-51.

25. Marchiori, LLM. Zumbido e hipertensão arterial no processo de envelhecimento. Rev Bras Hipertens. 2009; 16(1): 5-8.

26. Ferreira LMBM, Júnior ANR, Mendes EP. Caracterização do zumbido em idosos e de possíveis transtornos relacionados. Braz J Otorhinolaryngol. 2009; 75(2): 249-55.

27. Silva SSBE, Oliveira SFSB, Pierin AMG. O controle da hipertensão arterial em mulheres e homens: uma análise comparativa. Rev Esc Enferm USP. 2016; 50(1): 50-8.

28. Jastreboff PJ. Phantom auditory perception (tinnitus): mechanisms of generation and perception. Neurosci Res. 1990; 8: 221-54.

29. Branco- Barreiro FCAB, Knobel KAB, Sanchez TG. Modelo Neurofisiológico do Zumbido. In: Samelli AG. Zumbido, avaliação, diagnóstico e reabilitação. São Paulo: Lovise; 2004. p. 87-93.

30. Olzowy B, Canis M, Hempel JM, Mazurek B, Suckfüll M. Effect of Atorvastatin on Progression of Sensorineural Hearing Loss and Tinnitus in the Elderly: Results of a Prospective, Randomized, Double-Blind Clinical Trial. Otol Neurotol. 2007; 28: 455-8. 